degeneration, the transmitter disappears, even in cases in which the effective organ is not degenerated at all ; (2) on stimulation of the preganglionic cervical sympathetic the transmitter is liberated, not within the ganglion cell, but at the synapse-in other words, from the nerve-ending. Regarding the mechanism of the release we have to consider also two possibilities : either the transmitter is newly formed by the nerve-stimulation, or it is made diffusible, being split off from a combination already present in the nerve-ending. The decision between these alternatives cannot yet be made.

The lapse of time between the nerve-stimulation and the response of the reacting organ is extremely short, even in organs like the heart, where the transmitter has to pass a certain distance in order to reach the effector cells. The transmitters disappear somewhat quickly, the time of disappearance being de. pendent on the type of action which they have to produce.

The point of attack of the transmitters is not a part of the neurone, but the functioning, effective organ itself. The fact that the transmitters, when artificially injected, mainly act at points in relation to which they are normally liberated, can be given, as yet, only a teleological interpretation. Since the transmitters can diffuse into the blood from the point of their release, they can, in principle, also affect remote organs, though under physiological conditions this may never happen. Obviously such a distant action is unnecessary, as such needs are fulfilled by the hormones.

Finally, the question is considered whether the difference between the action of the hormones and that of the transmitters concerns only the sphere or also the character of their action. The hormones of two of the ductless glands, both being neurotropic and differing from all other ductless glands by properties common to them only - the adrenal medulla and the posterior lobe of the hypophysis-initiate or modify, according to need, the specific function of the organs, as the nerves or the transmitters do.

All the other ductless glands are not neurotropic ; they depend largely on the anterior lobe of the hypophysis regarding their development and state; their secretion is going on continuously and automatically, though partly controlled by the nervous system. Their action is concerned less with the specific functions of organs than with general conditions-state and metabolism. In other words, there are differences not only regarding the sphere but also regarding the character of the action of the nerves and the transmitters, on one hand, and that of the hormones on the other.

\title{
Cosmic Ray Results of the American Stratosphere Balloon Explorer I
}

$\mathrm{B}$ RIEF accounts have been given in NATURE ${ }^{1}$ of the flight of the American stratosphere balloon Explorer $I$ and the subsequent mishap by which it was at first feared that most of the valuable photographic records had been destroyed. Subsequent expert development of the films shows that, whilst the whole of the objectives have not been
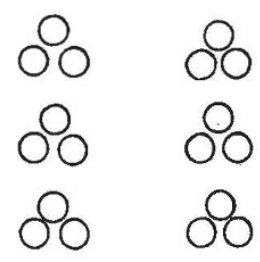

(a)

FIG. 1.

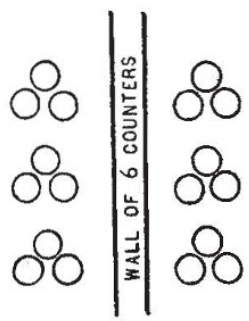

(b) counters dividing each bank. Apart from the influence of this wall on the time resolution, the arrangement of counters in Fig. $1 b$ would have the greater directive tendency. Two systems of recording were employed, one in which the total number of counts in a given time was integrated, and the other in which each individual count was recorded and timed. The curve of the spatial distribution of the rays at an altitude of $40,000 \mathrm{ft}$. for the two arrangements of the counters is shown in Fig. 2, in which the curve $a$ corresponds to the arrangement of Fig. $1 a$ and the

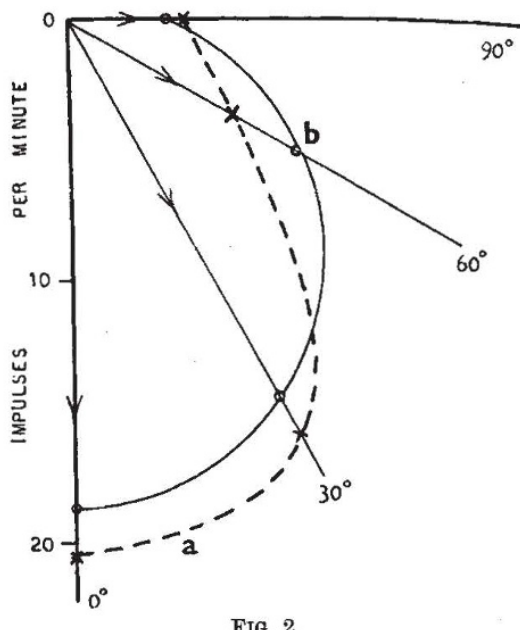

Fig. 2.

curve $b$ that in Fig. $1 b$. The number of counts varies from about 5 per minute for rays from the horizontal direction to about 20 per minute from the vertical at this height, whilst the more recent results from a flight by $J$. Piccard in America give similar curves up to $53,000 \mathrm{ft}$. The dotted curve $a$, which includes 
showers as well as individual rays, seems to show that the showers, on the whole, tend to come from the zenith.

When the average curve is integrated over a hemisphere and compared with a similar integration at sea-level, the ratio of the counter actions at the two heights is 64 . Millikan's result with a thin-walled ionisation chamber at $40,000 \mathrm{ft}$. is $J=250$ ions per c.c. per sec., so that if counter action is to be compared directly with volume ionisation by cosmic rays, the value of $J$ at sea-level would be $250 / 64=3.9$ ions per c.c. per sec. As against this value for $J$ three direct determinations are quoted, namely, Millikan $2 \cdot 4$, Compton $1 \cdot 9$, Hoffmann $1 \cdot 0$. Com. parison of the mean of these three figures with 3.9 indicates that counter action does not increase at the same rate with altitude as volume ionisation in a given locality. This is generally taken to mean that there is an increasing admixture of particles in higher altitudes that will not penetrate the counters but that will penetrate the walls of the ionisation chamber.

The Neher recording shielded electroscopes were destroyed in the crash, but an unshielded one gave a record up to $60,000 \mathrm{ft}$. The result for $J$ agrees with that of the Fordney Settle flight of November 1933.

2 Nature, 134, 132, July 28, 1934; 707, Nov. 3, 1934; 135, 299 , Feb. 23, 1935 .

\section{Surface Chemistry and its Industrial Applications}

$\mathrm{F}^{\mathrm{B}}$ industries are not dependent, at some stage in their processes, on reactions between matter in different states of aggregation, and consequently few industrial chemists can afford to ignore the recent developments in our knowledge of the physical and chemical changes that occur at surfaces. It is not surprising, therefore, that there was a record gathering for the discussion on the theoretical aspects and industrial applications of surface chemistry arranged by Section B for the Melbourne Centenary meeting of the Australian and New Zealand Association for the Advancement of Science. Prof. J. C. Earl was sectional president.

Dr. T. Iredale, opening the discussion, reviewed recent advances in the theory of surface chemistry, and other theoretical papers were presented by Dr. J. E. Mills on "The Recombination of Atoms and Free Radicles at Surfaces" and Dr. N. S. Bayliss on "A Critical Review of the Parachor". The contention that the parachor is of little value in determining the constitution of organic compounds was supported by Dr. R. A. Robinson. Mr. R. S. Burdon discussed the influence of carbon dioxide and hydrogen on the surface tension of mercury. Measurements of the amount of gas liberated by decreasing the surface area of a mercury surface prove that it adsorbs a unimolecular film that is tenaciously held, even in high vacuum. Prof. E. J. Hartung and the staff of the Chemistry Department of the University of Melbourne prepared a remarkably clear film of the Brownian movement in colloidal solutions. This film demonstrated the disintegration of benzopurpurin, the coagulation of copper ferrocyanide by sunlight, and the electrophoretic movement of the particles in either direction as the polarity of an applied electrical field was reversed. With a screen magnification of 40,000 , the translational, vibrational and rotational movements of colloidal particles were clearly seen.

Papers dealing with industrial applications of surface chemistry were delivered by Mr. C. Blazey on "The Effect of Service Conditions on Metal Surfaces", Mr. A. R. Hogg on "The Contact Process for Sulphuric Acid" and Mr. J. S. Wilson on "A Résumé of Current Theories of the Process of Dyeing Textile Fibres". No single theory of dyeing has yet been able to explain how the various types of dye are taken up silk, cotton and wool fibres.

At a joint discussion with Section H on "Corrosion", papers were delivered by Prof. J. N. Greenwood on "The Combined Influence of Stress and Corrosion on
Metals", Prof. A. Burn on "Cavitation in Turbines and Centrifugal Pumps", Dr. G. A. Elliott on "The Passivity of Metals in relation to Corrosion", Mr. P. F. Thompson on "Modern Theories of the Corrosion of Metals", Mr. C. J. Griffiths on "Electrolytic Corrosion" and Mr. V. Wardell on "Inhibitors of the Acid Corrosion of Metals". Mr. Wardell, discussing the cleaning of iron for galvanising, enumerated a great variety of substances that, though not preventing the removal of scale by acids, greatly reduce or even prevent the dissolution of iron. Glue is commonly used for this purpose. Generally known as inhibitors, these substances are used also for cleaning boilers and water mains. The addition to hydrochloric acid of a little arsenie chloride, while preventing dissolution of the steel base of galvanised iron, does not seriously retard the solution of the zinc coating.

Most of Australia's secondary industries, being still in the early stages of development, are forced to concentrate upon the solution of purely local problems. The base metal mining industry, however, grateful for benefits from the research work of other countries has, with the co-operation of the University of Melbourne, established a research laboratory for the investigation of the fundamental physico-chemical principles underlying the flotation process. Mr. H. Hey, opening a discussion on the chemistry of flotation, outlined its development from the laboratory stage, through the original large-scale operations at Broken Hill, to its present world-wide application to a great variety of ores. Mr. A. B. Cox and Dr. Ian W. Wark then described the work of the University laboratory. The flotation of a mineral depends upon its surface being modified so that it will adhere to an air bubble. Contact angle gives a quantitative measure of the condition of the surface. The influence of the adsorption of various organie compounds known as collectors on the magnitude of the angle of contact, and the influence of several inorganic compounds on their adsorption, have been determined. It is concluded that a unimolecular orientated adsorption of the collector is responsible for the attachment between mineral and air bubble. There is not yet agreement as to the mechanism of adsorption.

At a luncheon given by the Australian Chemical Institute, Dr. T. Callan conveyed greetings to the chemists of Australia from the Institute of Chemistry of Great Britain and Ireland and from the Society of Chemical Industry. 